\section{Mehr Aufmerksamkeit für Ultrafeinstaub}

Lanzinger S et al. Ultrafine and Fine Particles and Hospital Admissions in Central Europe. Results from the UFIREG Study. Am J Respir Crit Care Med 2016; 194: 1233 - 1241

Dass ein steigender Gehalt an Feinstaubpartikeln (Partikelgröße $<10 \mu \mathrm{m}$ $\left[\mathrm{PM}_{10}\right]$ und $<2,5 \mu \mathrm{m}\left[\mathrm{PM}_{2,5}\right]$ ) in der Luft zu mehr kardiologisch und respiratorisch bedingten Krankenhauseinweisungen führt, ist gut belegt. Im Rahmen der UFIREG-Studie wurde jetzt in 5 mittel- und osteuropäischen Städten auch der kurzfristige Effekt von ultrafeinen Partikeln (UFP) auf die ursachenspezifischen Krankenhauseinweisungen mituntersucht.

Aus Augsburg und Dresden konnten tägliche ursachenspezifische Krankenhauseinweisungen aus den Jahren 2011 und 2012 ausgewertet werden, aus Chernivtsi (Ukraine) aus den Jahren 2013 2014, aus Ljubljana (Slowenien) und Prag (Tschechische Republik) aus den Jahren 2012-2013. Primär interessierten die Forscher um Stefanie Lanzinger vom Helmholtz-Zentrum in München kardiovaskuläre (ICD-10: I00-199) und respiratorische Erkrankungen (ICD-10: J00J99), sie erfassten aber auch Einweisungen wegen Diabetes (ICD-10: E190-E14). Angaben zur Luftverschmutzung und metereologische Daten stammten aus fixen Messstationen der 5 Städte. Die UFPBelastung war in allen Städten nur mäßig mit den $\mathrm{PM}_{2,5^{-}}$und $\mathrm{PM}_{10}$-Werten korreliert.

\section{Ergebnisse}

Der mittlere Interquartilsabstand lag bei 2.750 UFP $/ \mathrm{cm}^{3}$. Stieg der UFP im 6-Tages-Mittel um diese Konzentration an, war das ein Indikator für einen verzögerten und verlängerten Anstieg des gepoolten relativen Risikos (RR) für Krankenhauseinweisungen aufgrund von Atemwegerkrankungen um 3,4\% (95\% Konfidenzintervall $-1,7$ bis $8,8 \%$ ). Nur über 2 Tage betrachtet war der Effekt eines UFP-Anstiegs auf Krankenhauseinweisungen dagegen deutlich geringer. Eine

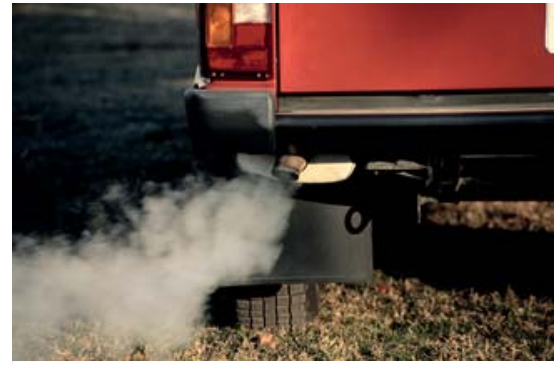

Als ultrafeine Partikel (UFP oder PM0,1) werden Partikel bezeichnet, deren aerodynamischer Durchmesser kleiner als 0,1 Mikrometer oder kleiner als $100 \mathrm{Na}$ nometer ist. Bildquelle: PhotoDisc

besonders ausgeprägte Assoziation zeigte sich in Augsburg und Dresden, wo auch insgesamt die meisten Krankenhauseinweisungen in den betrachteten Indikationen registriert worden waren.

Den bereits vielfach beschriebenen Anstieg kardiovaskulär und respiratorisch bedingter Krankenhausaufnahmen bei einem Anstieg der Belastung mit $\mathrm{PM}_{2,5}$ fanden die Autoren ebenfalls, außerdem einen Anstieg der diabetesbedingten Einweisungen in Abhängigkeit sowohl von UFP als auch $\mathrm{PM}_{2,5}$ und $\mathrm{PM}_{10}$. Zudem nahmen atemwegsbedingte Krankenhauseinweisungen auch abhängig von der $\mathrm{NO}_{2}$-Exposition zu.

FAZIT

Der Einfluss von UFP auf Krankenhauseinweisungen aufgrund von kardiovaskulären oder respiratorischen Diagnosen unabhängig von größeren Feinstaubpartikeln ist in verschiedenen Regionen Europas festzustellen. Daher plädieren die Autoren dafür, UFP in das regelhafte Monitoring der Luftverschmutzung aufzunehmen und die kurz-, mittel- und langfristigen gesundheitlichen Folgen der UFP-Belastung weiter zu erforschen. Dazu sollten die Messmethoden europaweit harmonisiert werden.

Friederike Klein, München 